and radiopacity in a water-filled phantom. Improved kink radii compared to a commercial catheter demonstrated a device that could maintain lumen patency during navigation. Device construction featured simple manufacturing steps to produce a low-profile catheter with MRI and X-ray visibility for neurointerventional applications.

Disclosures B. Kilbride: 6; C; Penumbra, Inc. C. Jordan: 6; C; Penumbra, Inc. A. Chu: None. D. Barry: None. T. Moore: None. A. Martin: None. M. Wilson: None. S. Hetts: None.

\section{E-104 IMAGE GUIDANCE FOR MECHANICAL THROMBECTOMY IN STROKE USING AN OPTICAL SEE-THROUGH HEAD- MOUNTED DISPLAY (OST-HMD): PROOF OF CONCEPT AND RATIONALE}

${ }^{1} \mathrm{G}$ Deib*, ${ }^{2} \mathrm{~T}$ Song, ${ }^{2} \mathrm{~W}$ Gu, ${ }^{3}$ J Perkowski, ${ }^{2} \mathrm{~N}$ Navab, ${ }^{2} \mathrm{M}$ Unberath, ${ }^{4} \mathrm{~F}$ Hui, ${ }^{4} \mathrm{P}$ Gailloud. ${ }^{1}$ Neuroradiology, West Virginia University, Morgantown, WVi ${ }^{2}$ Computer Aided Medical Procedures, Johns Hopkins University, Baltimore, MD; ${ }^{3}$ Cerenovus, Irvine, $C A$; ${ }^{4}$ Neuroradiology, Johns Hopkins University, Baltimore, MD

\subsection{6/neurintsurg-2020-SNIS.137}

Background and Purpose Optical see-through head-mounted displays (OST-HMD) can enable a mixed reality (MR) experience for neurointerventionalists during procedures encompassing high resolution radiographic imaging and an unhindered view of the procedural site. The authors present a technical note detailing an approach to mechanical thrombectomy in stroke utilizing an OST-HMD as an alternative to traditional angiography suite display monitors.

Methods Mixed reality visualization was achieved using the Microsoft HoloLens system. An anatomically realistic flow model was employed to perform the procedure. A commercially available guide sheath, intermediate aspiration catheter, microcatheter and mechanical thrombectomy device were utilized to perform a mechanical thrombectomy of a right M1 thrombus. The head mounted display created a real-time mixed reality environment by superimposing the virtual AP and lateral views onto the interventionalist's field of view. The procedure was filmed through the point of view of the operator. The video was reviewed to assess whether key anatomic landmarks and materials could be consistently and reliably visualized. Dosimetry and time of procedure were recorded. The operator completed a questionnaire following the

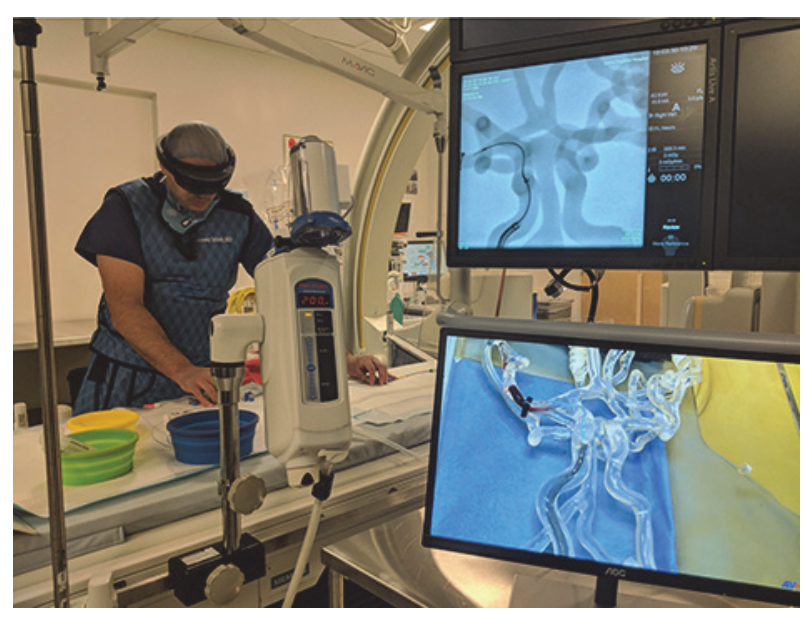

Abstract E-104 Figure 1 procedure detailing benefits, limitations, and visualization mode preferences.

Results A right M1 thrombectomy was successfully performed using OST-HMD image guidance on an anatomically realistic flow model. Dosimetry and procedural time compared favorably to typical procedural times. All visualization modes were equally effective in providing image guidance. Key anatomic landmarks and materials were consistently and reliably visualized.

Conclusions This preliminary study demonstrates that mechanical thrombectomy for stroke utilizing OST-HMDs for image guidance is feasible. This novel visualization approach may serve as a valuable tool for performing mechanical thrombectomy and other endovascular imageguided procedures.

Disclosures G. Deib: None. T. Song: None. W. Gu: None. J. Perkowski: None. N. Navab: None. M. Unberath: None. F. Hui: None. P. Gailloud: None.

\section{E-105 LEARNING CURVE FOR DIAGNOSTIC CEREBRAL ANGIOGRAPHY: TRANSRADIAL ACCESS VERSUS TRANSFEMORAL ACCESS}

M Tso*, G Rajah, R Dossani, M Meyer, M McPheeters, K Vakharia, M Waqas, K Snyder, E Levy, A Siddiqui, J Davies. Neurosurgery, University at Buffalo, Buffalo, NY

\subsection{6/neurintsurg-2020-SNIS. 138}

Background Diagnostic cerebral angiography (DSA) and neurointerventions have traditionally been performed via transfemoral access (TFA). The perception of a steep learning curve associated with transradial access (TRA) has limited adoption in neurointervention. This study compares the learning curves of transradial vs. transfemoral DSA in a cohort of neurointerventional fellows.

Methods The first 150 consecutive radial and femoral DSA were identified for each fellow from a prospective neurointerventional registry from July 2017 to March 2020. Total fluoroscopy time and number of intracranial arteries injected were recorded. Mean fluoroscopy time per intracranial artery injected (termed angiographic efficiency) was calculated and was used as a surrogate measure of technical proficiency. Mean angiographic efficiencies were compared across partitions of 25 consecutive DSAs (e.g. 1-25, 26-50, $51-75$, etc.).

Results There were 607 radial DSA and 635 femoral DSA identified among 5 fellows. The overall angiographic efficiencies were not significantly different based on access site (radial mean $3.2 \mathrm{~min}$, femoral mean $3.7 \mathrm{~min}, \mathrm{p}>0.05$ ). For 3 fellows without prior endovascular experience, technical proficiency was obtained between 25-50 femoral DSA procedures. Among these same fellows, one fellow achieved technical proficiency after 25-50 radial DSA procedures, while the other 2 fellows had flattened learning curves. There were 2 fellows that had no significant learning curve for either access type, but both had extensive experience with endovascular procedures prior to starting fellowship. Two patients $(2 / 1342=0.1 \%)$ experienced transient neurologic symptoms post-procedure. Among 635 femoral DSA, there were 22 (3.5\%) minor adverse events (14 small groin hematomas not requiring transfusion, 1 pseudoaneurysm, 7 non-flow-limiting dissections). Among 607 radial DSA, there were $3(0.5 \%)$ minor adverse events (2 small forearm hematomas, 1 intraluminal wire removed with 
radial cut-down), which was significantly less than femoral DSA minor adverse events $(p=0.0001)$. Radial to femoral conversion rate was $1.2 \%(7 / 607,2$ radial spasm, 2 unsuccessful radial artery access, 3 aberrant right subclavian artery). Femoral to radial conversion rate was 0.3\% (2/635). From March 2019 to February 2020, the proportion of DSA performed via transradial access increased from $36 \%$ to $78 \%$ at our institution.

Conclusion This study demonstrates the learning curves for both TRA and TFA diagnostic cerebral angiograms. Proficiency improves significantly with either type of access, typically requiring between 25-50 procedures. TRA DSA have significantly less access site complications than TFA DSA.

Disclosures M. Tso: None. G. Rajah: None. R. Dossani: None. M. Meyer: None. M. McPheeters: None. K. Vakharia: None. M. Waqas: None. K. Snyder: None. E. Levy: None. A. Siddiqui: None. J. Davies: None.

\section{E-106 EFFICACY OF ASAHI FUBUKI AS A GUIDING CATHETER FOR MECHANICAL THROMBECTOMY: AN INSTITUTIONAL CASE SERIES}

${ }^{1} \mathrm{~L}$ Rinaldo*, ${ }^{2} \mathrm{H}$ Cloft, ${ }^{3} \mathrm{~W}$ Brinjikji. ${ }^{1}$ Neurosurgery, Mayo Clinic, Rochester, MN; ${ }^{2}$ Radiology, Mayo Clinic, Rochester, MN; ${ }^{3}$ Radiology and Neurosurgery, Mayo Clinic, Rochester, MN

\subsection{6/neurintsurg-2020-SNIS.139}

Background Delivery of a guiding catheter to a suitable position is a critical step in performing mechanical thrombectomy (MT) for treatment of acute stroke secondary to large vessel occlusion. Over the past several years, we have employed the Asahi Fubuki (Asahi Intecc Co., LTD, 1976) as a first-line guide catheter for the performance of MT. Herein, we reviewed the treatment outcomes of MT cases in which the Fubuki was utilized to assess the efficacy of this catheter.

Methods Radiology reports of MT for the treatment of acute stroke secondary to large vessel occlusion during which the Fubuki was employed as a guide catheter were retrospectively reviewed. Information on patient characteristics and treatment outcomes was collected. Predictors of failure to deliver the Fubuki to the cervical internal carotid artery (ICA) were identified using logistic regression analysis.

Results There were 124 patients who met the inclusion criteria for analysis. Mean patient age was 77.8 years and a slight majority of patients were female (50.8\%). Large vessel occlusion occurred most often at the M1 segment $(41.1 \%)$ and most occlusions occurred on the patient's right side $(57.5 \%)$. The Fubuki was successfully delivered to the cervical ICA in 121 cases (97.6\%), and the lesion was successfully crossed with the Fubuki as the guide catheter in 119 cases (96.0\%). Herniation of the Fubuki into the aortic arch after delivery to the cervical ICA and cross-over to another guide catheter each occurred once $(0.8 \%)$ in separate cases. Greater than or equal to TICI $2 \mathrm{~B}$ revascularization was achieved in 100 patients $(80.6 \%)$. Median times to lesion crossed and revascularization were 9 and 19 minutes.

Conclusion Our results indicate that the Asahi Fubuki is an effective guide catheter for the performance of MT.

Disclosures L. Rinaldo: None. H. Cloft: None. W. Brinjikji: None.

\section{E-107 PREDICTORS OF DECOMPRESSIVE HEMICRANIECTOMY IN SUCCESSFULLY RECANALIZED PATIENTS WITH ANTERIOR CIRCULATION EMERGENCY LARGE VESSEL OCCLUSION}

${ }^{1} \mathrm{~N}$ Goyal ${ }^{*},{ }^{2} \mathrm{G}$ Tsivgoulis, ${ }^{2} \mathrm{~A}$ Pandhi, ${ }^{2} \mathrm{M}$ Ishfaq, ${ }^{2} \mathrm{~J}$ Goyanes, ${ }^{2} \mathrm{~A}$ Deep, ${ }^{2} \mathrm{D}$ Alsbrook, ${ }^{2} \mathrm{~S}$ Singh, ${ }^{2} \mathrm{G}$ Zaid, ${ }^{3} \mathrm{D}$ Hoit, ${ }^{3} \mathrm{C}$ Nickele, ${ }^{2} \mathrm{~V}$ Inoa, ${ }^{3} \mathrm{D}$ Dornbos, ${ }^{2} \mathrm{~A}$ Alexandrov, ${ }^{3} \mathrm{~A}$ Arthur, ${ }^{1} \mathrm{~L}$ Elijovich. ${ }^{1}$ Neurology and Neurosurgery, UTHSC, Memphis, TN; ${ }^{2}$ Neurology, UTHSC, Memphis, TN; ${ }^{3}$ Neurosurgery, UTHSC, Memphis, TN

\subsection{6/neurintsurg-2020-SNIS.140}

Background and Purpose Mechanical thrombectomy (MT) is demonstrated to improve functional outcome of patients with anterior circulation strokes with emergency large vessel occlusion (ELVO). Despite successful recanalization (SR), a proportion of these patients require decompressive hemicraniectomy (DHC). We aimed to study the predictors of DHC in successfully recanalized anterior circulation ELVO patients.

Methods Consecutive anterior circulation ELVO patients treated with MT during a 6-year period in a tertiary stroke center were evaluated. Only patients with SR (mTICI 2b, 2c or 3) after MT were included in the analysis. Baseline demographic, clinical and procedural variables were compared between the patients who required DHC after successful recanalization vs. who did not. Collaterals for anterior circulation ELVO (ie, poor (collateral score=0) vs. good (collateral score $=1,2$ and 3) were reported using a methodology that has been shown to predict clinical outcome. Good functional outcome was defined as mRS score of $0-2$ at 3 months. Univariable and multivariable logistic regression analyses were used to determine the predictors of DHC.

Results Of 453 successfully recanalized anterior circulation

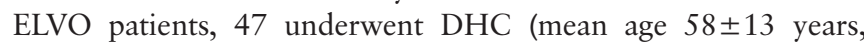
53\% male, median admission NIHSS-score: 17 points, IQR 13-21). The patients in DHC group were younger in age (mean age in years \pm SD; $58 \pm 13$ vs. $65 \pm 15, \mathrm{p}=0.004$ ), had higher admission systolic blood pressure (mmHg: $167 \pm 33$ vs. $155 \pm 30, \mathrm{p}=0.010)$, higher admission diastolic blood pressure (mmHg: $95 \pm 28$ vs. $87 \pm 19, \mathrm{p}=0.019$ ), higher admission blood glucose levels (mg/dl: $170 \pm 88$ vs. $142 \pm 66, \mathrm{p}=0.008$ ), similar median admission NIHSS (17 (IQR: 13-21) vs. 15 (IQR: 1120), $\mathrm{p}=0.087$ ), lower median ASPECTS (9 (IQR: 8-10) vs. 10 (IQR: 9-10), $\mathrm{p}=0.002)$, and poor collaterals on pre-treatment CT angiogram (\% with poor collaterals: $75 \%$ vs. $26 \%$, $\mathrm{p}<0.001)$ compared to those who did not required DHC. The DHC group required more passes during MT (median: 3 (IQR: 3-4) vs. 2 (IQR 1-2), p=0.001), had longer groin puncture to recanalization time (mean: $74 \pm 32$ vs. $55 \pm 35$, $\mathrm{p}<0.001$ ), while no difference was seen in symptoms onset to groin puncture time (mean: $313 \pm 166$ vs. $304 \pm 202, p=0.784$ ) compared to the non-DHC group. The patient in DHC group tended to have a higher $\mathrm{sICH}$ rate $(15 \%$ vs. $7 \%, \mathrm{p}=0.20)$, higher 3 months mortality $(41 \%$ vs. $16 \%, p=0.001)$ and lower rates of good functional outcome at 3 months $(26 \%$ vs. $58 \%, \mathrm{p}=0.001)$. In multivariable model after adjusting for various confounders, higher blood glucose levels on admission (OR per $1 \mathrm{mg} / \mathrm{dL}$ increase: 1.01; 95\% CI: 1.00-1.01, $\mathrm{p}=0.031$ ), poor collaterals on CT angiography (OR: 0.13 ; 95\% CI: $0.05-0.32, \mathrm{p}<0.001)$, and higher number of passes during MT (OR per 1 pass increase: 4.84; 95\% CI: 3.09$7.58, \mathrm{p}<0.001$ ) emerged as independent predictors of $\mathrm{DHC}$ in successfully recanalized ELVO patients.

Conclusion Higher blood glucose levels on admission, poor collateral pattern on CT angiography and higher number of 